\title{
Difficult Laparoscopic Cholecystectomy and Trainees: Predictors and Results in an Academic Teaching Hospital
}

\author{
Hussein M. Atta, Ashraf A. Mohamed, Alaa M. Sewefy, Abdel-Fatah S. Abdel-Fatah, \\ Mohammed M. Mohammed, and Ahmed M. Atiya
}

Department of General Surgery, Faculty of Medicine, Minia University, El-Minia 61519, Egypt

Correspondence should be addressed to Hussein M. Atta; atta@mu.edu.eg

Received 30 March 2017; Accepted 22 May 2017; Published 5 June 2017

Academic Editor: Piero Chirletti

Copyright (c) 2017 Hussein M. Atta et al. This is an open access article distributed under the Creative Commons Attribution License, which permits unrestricted use, distribution, and reproduction in any medium, provided the original work is properly cited.

\begin{abstract}
Laparoscopic cholecystectomy (LC) is one of the first laparoscopic procedures performed by surgical trainees. This study aims to determine preoperative and/or intraoperative predictors of difficult LC and to compare complications of LC performed by trainees with that performed by trained surgeons. A cohort of 180 consecutive patients with cholelithiasis who underwent LC was analyzed. We used univariate and binary logistic regression analyses to predict factors associated with difficult LC. We compared the rate of complications of LCs performed by trainees and that performed by trained surgeons using Pearson's chi-square test. Patients with impacted stone in the neck of the gallbladder (GB) (OR, 5.0; 95\% CI, 1.59-15.77), with adhesions in the Triangle of Calot (OR, 2.9; 95\% CI, 1.27-6.83), or with GB rupture (OR, 3.4; 95\% CI, 1.02-11.41) were more likely to experience difficult LC. There was no difference between trainees and trained surgeons in the rate of cystic artery injury ( $p=.144)$ or GB rupture $(p=.097)$. However, operative time of LCs performed by trained surgeons was significantly shorter (median, $45 \mathrm{~min}$; IQR, 30-70 min) compared with the surgical trainees' operative time (60 min; IQR, 50-90 min). Surgical trainees can perform difficult LC safely under supervision with no increase in complications albeit with mild increase in operative time.
\end{abstract}

\section{Introduction}

Laparoscopic cholecystectomy (LC) is the standard of care for patients with cholelithiasis. Several randomized controlled trials and systematic reviews have demonstrated the effectiveness and safety of LC for the treatment of symptomatic cholelithiasis [1-4]. The rapid acceptance of LC as the standard of care for patients with gallstones has been attributed to several benefits including decreased patient morbidity, faster recovery, and shorter hospital stay when compared to open cholecystectomy [5-7].

LC is one of the first laparoscopic procedures performed by surgical trainees. Despite the establishment of formal training in laparoscopic surgery and the improvement in laparoscopic technology, still, there is a perception that performance of LCs in teaching hospitals with continuous inflow of trainees may be attended with difficult LC, increased conversion, and complication rates [8]. While several studies have reported a variable assembly of different preoperative and operative risk factors associated with difficult LC and conversion to open cholecystectomy [9-13], the performance of surgical trainees with different training backgrounds has not been adequately addressed.

Although conversion of LC to open cholecystectomy is considered an important outcome of LC, however, currently, conversion rate is less common $(2.6 \%-5.2 \%)$ than other surrogate parameters of difficult LC such as operative time more than $60 \mathrm{~min}$, adhesions in the Triangle of Calot, cystic artery injury, or spillage of stones [14-16].

This study is conducted to determine predictors of difficult LC, defined as operative time more than $60 \mathrm{~min}$ and/ or cystic artery injury, in the setting of a single academic teaching hospital and in particular to compare the outcomes of LCs performed by surgical trainees with those performed by trained surgeons. 


\section{Methods}

This retrospective cohort included 180 consecutive patients with cholelithiasis who underwent LC at Minia University Hospital, El-Minia, Egypt, from November 2014 to October 2016. The study protocol was approved by the Faculty of Medicine Minia University Council. Informed consent was obtained from all patients, and data were collected prospectively. In order to have a homogenous patient population, we excluded patients with acute cholecystitis, pancreatitis, common bile duct (CBD) stone, and those who underwent combined LC with any other laparoscopic interventions including laparoscopic CBD exploration. All LCs were performed on an elective basis. LCs were performed by surgeons with three years of general surgery training and are referred to as surgical trainees or by experienced laparoscopic surgeons who had more than five years of surgery training and are referred to as trained surgeons. During their training, surgical trainees assisted in at least 150 LCs but did not assume the role of surgeon, while trained surgeons had performed more than 25 unsupervised LCs [17].

Both groups were assigned to LCs according to their duty schedule; thereby, no surgeon selection was attempted. However, surgical trainees were supervised in the theater by a nonscrub trained surgeon. All LC procedures were completed by the initial operating surgeon. Thus, this study setting reflects a real setting of a midsize university teaching hospital. LCs were performed either with the retrograde approach (dissection initiated from the Triangle of Calot upward to the fundus of the gallbladder) or with the domedown technique (removing the gallbladder from the gallbladder bed first) according to the surgeon discretion in lieu of the severity of adhesions at the Triangle of Calot. Retrograde approach was used when there are minimal, easily dissectible adhesions, while dome-down technique was used in the presence of severe adhesions. Difficult LCs were defined as LC with operative time of more than $60 \mathrm{~min}$, or with injury to the cystic artery before ligation or clipping [16]. Patients' characteristics including demographic, clinical, ultrasonographic, and operative parameters that could contribute to predicting operative difficulties were analyzed.

2.1. Statistical Analysis. Categorical variables are presented as counts and percentages. Continuous variables are presented as mean \pm standard deviation or median (25th-75th interquartile range, IQR) for normally or not normally distributed variables, respectively. Shapiro-Wilk test was used to test for a normal distribution. Univariate analysis of patients' characteristics was performed to identify variables associated with difficult LC. Categorical variables were compared using Fisher exact test, and continuous variables not normally distributed were compared using the nonparametric Mann-Whitney $U$ test. To identify independent predictors of difficult LC, variables with a $p$ value $<0.05$ were subsequently entered into a binary logistic regression model [18]. Validity of the model was checked using the Hosmer and Lemeshow goodness of fit test [19]. The difference between the rate of complications of LCs performed by surgical trainees and that performed by trained surgeons was compared using Pearson's chi-square or Mann-Whitney $U$ test. For all statistical analyses, two-tailed tests were used. Statistical analysis was performed using the software Statistical Package for Social Sciences, SPSS version 13 (SPSS, Chicago, IL, USA). A $p$ value of $<0.05$ was considered statistically significant.

\section{Results}

3.1. Patient Characteristics. A total of 180 consecutive patients underwent LC at Minia University Teaching Hospital from November 2014 to October 2016. Fifty eight LCs $(32 \%)$ fulfilled the criteria of difficult LC defined as operative time of more than $60 \mathrm{~min}$ or injury to the cystic artery. Coronary heart disease, hemolytic anemia, and hepatitis $C$ virus infection each occurred in a single patient and were not entered in the analysis. Twenty three per cent of LCs was performed by surgical trainees. Cystic artery injury occurred in six LCs, and there was no CBD injury. A single LC was converted to open cholecystectomy in this cohort due to inability to control bleeding from injured cystic artery.

3.1.1. Risk Factors for Difficult LC. Comparison of patient characteristics between difficult and easy LCs identified nine risk factors for difficult LC that differed significantly (Table 1). Injury of the cystic artery and its related blood loss $>50 \mathrm{~mL}$ and operative time $\geq 60 \mathrm{~min}$ were not included in the regression analysis because they constitute the definition of difficult LC. Identified risk factors include, male gender, gallbladder (GB) wall thickness $\geq 4 \mathrm{~mm}$, GB fluid containing sludge, impacted stone in the neck of the GB, pericholecystic fluid collection, adhesions in the Triangle of Calot, ruptured GB, spilled stones, and surgeon skill of less than ten LCs.

3.1.2. Regression Model Performance. A binary logistic regression analysis was performed to determine the effects of risk factors on the likelihood that patients having difficult LC. The binary logistic regression model was statistically significant, $\chi^{2}=67.202, p<.001$. The Hosmer and Lemeshow goodness of fit test suggests that the model is a good fit to the data as $p=0.460$ is nonsignificant [19]. The model explained $43.5 \%$ (Nagelkerke $R^{2}$ ) of the variance in difficult LC. The classification table (Table 2) is a method to evaluate the predictive accuracy of the logistic regression model. In this table, the observed values for the dependent outcome and the predicted values (at a cutoff value of $p=0.50$ ) are cross-classified. Our model correctly predicts $81.7 \%$ of cases. We calculated the error rates from the classification table output. A false positive would be predicting that difficult LC would occur when, in fact, it did not. Our model predicted difficult LC 43 times. That prediction was wrong nine times, for a false positive rate of $9 / 43=20.9 \%$. A false negative would be predicting that difficult LC would not occur when, in fact, it did occur. Our model predicted difficult LC not to occur in 137 times. That prediction was wrong 24 times, for a false negative rate of $24 / 137=17.5 \%$.

3.2. Predictors of Difficult LC. This model suggests that impacted stone in the neck of the GB, adhesions in the 
TABLE 1: Comparison of patient characteristics between difficult and easy LCs.

\begin{tabular}{|c|c|c|c|}
\hline Characteristics & $\begin{array}{l}\text { Difficult LC } \\
\quad(n=58)\end{array}$ & $\begin{array}{l}\text { Easy LC } \\
(n=122)\end{array}$ & $p$ value \\
\hline \multicolumn{4}{|l|}{ Preoperative characteristics } \\
\hline Male gender ${ }^{\dagger}$ & $16(27.6 \%)$ & $17(13.9 \%)$ & 0.038 \\
\hline Age $>65$ years & $1(1.7 \%)$ & $4(3.3 \%)$ & 1.000 \\
\hline BMI, $\geq 30 \mathrm{~kg} / \mathrm{m}^{2}$ & $10(17.2 \%)$ & $19(15.5 \%)$ & 0.829 \\
\hline Smoking & $6(8.6 \%)$ & $5(4.1 \%)$ & 0.179 \\
\hline Elevated liver enzymes & $1(1.7 \%)$ & $1(0.8 \%)$ & 0.542 \\
\hline Previous abdominal operation & $12(20.1 \%)$ & $21(17.2 \%)$ & 0.681 \\
\hline Hypertension & $5(8.6 \%)$ & $8(6.6 \%)$ & 0.412 \\
\hline Diabetes mellitus & $3(5.2 \%)$ & $2(1.6 \%)$ & 0.330 \\
\hline Liver cirrhosis & $3(5.2 \%)$ & $2(1.6 \%)$ & 0.330 \\
\hline Previous biliary hospitalization & $13(22.4 \%)$ & $18(14.8 \%)$ & 0.212 \\
\hline Palpable GB & 0 & $3(2.5 \%)$ & 0.552 \\
\hline GB wall thickness, $\geq 4 \mathrm{~mm}^{\dagger}$ & $38(65.5 \%)$ & $49(40.2 \%)$ & 0.002 \\
\hline $\begin{array}{l}\text { GB transverse diameter, } \\
<2,>5 \mathrm{~cm}\end{array}$ & $18(31.0 \%)$ & $40(32.8 \%)$ & 0.866 \\
\hline GB sludge $^{\dagger}$ & $32(55.2 \%)$ & $22(18 \%)$ & 0.000 \\
\hline $\begin{array}{l}\text { Impacted stone in the } \\
\text { neck of } \mathrm{GB}^{\dagger}\end{array}$ & $23(39.7 \%)$ & $7(5.7 \%)$ & 0.000 \\
\hline Pericholecystic fluid collection $^{\dagger}$ & $5(8.6 \%)$ & $1(0.82 \%)$ & 0.014 \\
\hline CBD diameter, $>10 \mathrm{~mm}$ & $2(3.4 \%)$ & $4(3.3 \%)$ & 1.000 \\
\hline CBD stones & $1(1.7 \%)$ & $1(0.82 \%)$ & 0.542 \\
\hline Surgeon LC skill, $<10 \mathrm{LCs}^{\dagger}$ & $19(32.8 \%)$ & $22(18 \%)$ & 0.036 \\
\hline Surgeon LC skill, >25 LCs & $26(44.8 \%)$ & $75(61.5 \%)$ & 0.028 \\
\hline \multicolumn{4}{|l|}{ Intraoperative characteristics } \\
\hline Operative time, $>60 \mathrm{~min}$ & $57(98.3 \%)$ & $40(32.8 \%)$ & 0.000 \\
\hline Operative blood loss, $>50 \mathrm{~mL}$ & $30(51.7 \%)$ & $29(23.8 \%)$ & 0.000 \\
\hline Triangle of Calot adhesions ${ }^{\dagger}$ & $36(62.1 \%)$ & $29(23.7 \%)$ & 0.000 \\
\hline Ruptured $\mathrm{GB}^{\dagger}$ & $29(50 \%)$ & $19(15.6 \%)$ & 0.000 \\
\hline Spilled stones ${ }^{\dagger}$ & $20(34.5 \%)$ & $10(8.2 \%)$ & 0.000 \\
\hline Cystic artery injury & $6(10.3 \%)$ & 0 & 0.001 \\
\hline CBD injury & 0 & 0 & 0 \\
\hline Conversion & $1(1.7 \%)$ & 0 & 0.322 \\
\hline
\end{tabular}

${ }^{\dagger}$ characteristics included in binary logistic regression analysis.

Triangle of Calot, and GB rupture during LC are independent predictors of difficult LC (Table 3). Patients with impacted stone in the neck of the GB are about five times (odds ratio $[\mathrm{OR}], 5.0$; 95\% confidence interval [CI], 1.5915.77) likely to undergo a difficult LC. This model also shows that patients with adhesions in the Triangle of Calot (OR, 2.9; 95\% CI, 1.27-6.83) or with GB rupture during LC (OR, 3.4; 95\% CI, 1.02-11.41) are about three times more likely to experience difficult LC (Table 3).

3.3. Outcome of Trainee-Performed LCs. Although our regression model did not select trainees as a predictor of difficult LC, however, we hypothesized that there may be a difference between the rate of complications of LCs performed by trainees with experience of less than ten LCs and that performed by trained surgeons with skills of more than
TABle 2: Classification table.

\begin{tabular}{lccc}
\hline \multirow{2}{*}{ Observed } & \multicolumn{3}{c}{ Predicted } \\
& Easy & Difficult & Percentage correct \\
\hline Easy & 113 & 9 & 92.6 \\
Difficult & 24 & 34 & 58.6 \\
Overall percentage & & & 81.7 \\
\hline
\end{tabular}

25 unsupervised LCs. We found that there is no statistically significant difference between trainees and trained surgeons in the rate of cystic artery injury $(4.9 \%$ and $1.0 \%$, Pearson's chi-square, $p=0.144)$ or GB rupture $(17.1 \%$ and $30.7 \%$, $p=0.097$ ). As expected, we found that operative time of LCs performed by trained surgeons was significantly shorter (median, $45 \mathrm{~min}$; IQR, 30-70 min) compared with surgical trainees' operative time (60 min; IQR, 50-90 min) (Mann-Whitney $U$ test, $p=0.001$ ) (Figure 1 ).

\section{Discussion}

This study suggested that impacted stone in the neck of the GB, the presence of adhesions in the Triangle of Calot, GB rupture, and injury to the cystic artery predicted increase in the likelihood of having difficult LC. Furthermore, we showed also that in case of difficult LC performed by surgical trainees under direct supervision of trained surgeons, there was no increase in the LC complications, cystic artery injury, GB rupture, or conversion when compared with trained surgeons. There is, however, infrequent increase in the operative time of LCs performed by surgical trainees.

Currently, LC is the standard of care for patients with cholelithiasis and is the first laparoscopic surgical procedure to be performed by general surgery trainees in many teaching hospitals [20]. These laparoscopic skills must be passed on to junior surgeons without compromising patient safety. In our surgical training program, we do not use surgical simulators or cadaveric surgery for laparoscopic surgery training but we solely rely on extended operative assistance. Our surgical trainees start performing LC only after assisting in at least 150 LCs during their previous three years of surgical training. This study showed also that surgical trainees, who performed LCs under direct supervision of trained surgeons, had no increase in the LC complications when compared with trained surgeons. However, the operative time is longer in LCs performed by surgical trainees compared with trained surgeons. In agreement with our results, Lavy et al. reported a comparative study of LC performed by residents with that performed by senior surgeons [20]. They found that the only significant difference between the groups was a longer operative time, while the conversion rate and complication rate were the same. In a similar study comparing consultant surgeons, trainee surgeons, and trained surgeons, the authors found that there were no differences among the three groups in conversion rates, bile duct injury rates, general complication rates, or length of stay; however, the duration of operation in the trainee surgeons was significantly longer compared to the other two groups [21]. In the setting of 
TABLE 3: Binary logistic regressions analysis of risk factors for difficult LC.

\begin{tabular}{lccccc}
\hline & Regression coefficient & Wald statistic & $p$ value & Odds ratio & 95\% C.I. \\
\hline Impacted stone & 1.614 & 7.628 & .006 & 5.021 & 1.598 \\
Calot's adhesions & 1.079 & 6.305 & .012 & 2.943 & 15.779 \\
GB rupture & 1.225 & 3.943 & .047 & 3.405 & 6.834 \\
\hline
\end{tabular}

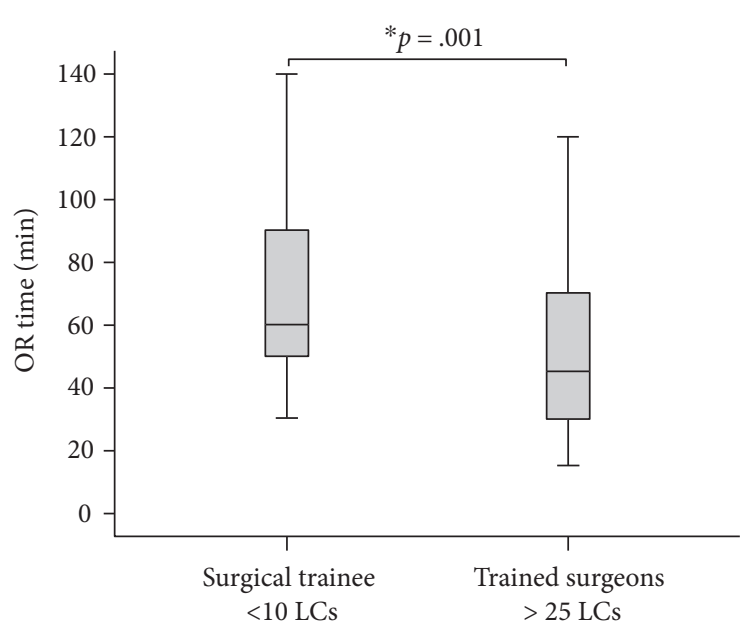

FIGURE 1: Boxplots (median, interquartile range, max, and min) of LC operative time of surgical trainees and trained surgeons (min). *significant differences between surgical trainees and trained surgeons.

LC for acute cholecystitis, Abelson et al. reported that advanced laparoscopic fellowship-trained surgeons had significantly lower conversion rate and shorter operative time than the nonfellowship-trained surgeons; however, the complication rates were not significantly different [22].

The low incidence of conversions in our cohort of 180 consecutive patients with gall stone disease is primarily due to the fact that this series did not include LC performed in patients with acute cholecystitis, pancreatitis, or CBD stone. A study from a single university medical center reported a conversion rate of $2.6 \%$, and the diagnosis of acute cholecystitis was more common among converted cases [15]. In a recent analysis of preoperative risk factors for conversion from a prospective U.K. database of 8820 patients, Sutcliffe et al. reported a rate of conversion when the indication for LC was for cholecystitis $(6.5 \%)$ to be higher than that for colic $(1.2 \%)$ or for pancreatitis $(2.1 \%)$ but only lower than that of $\mathrm{CBD}$ stone $(9.1 \%)[23]$.

\section{Conclusion}

This study demonstrated that operative complications of LC performed by surgical trainees who had extended operative exposure and who performed LC under direct supervision of trained surgeons are not different from those performed by trained surgeons except in moderate increase of operative time.

\section{Ethical Approval}

The study protocol was approved by the Faculty of Medicine Minia University Council, and informed consent was obtained from all patients.

\section{Conflicts of Interest}

The authors declare that there is no conflict of interest regarding the publication of this article.

\section{References}

[1] W. Ji, L. T. Li, Z. M. Wang, Z. F. Quan, X. R. Chen, and J. S. Li, "A randomized controlled trial of laparoscopic versus open cholecystectomy in patients with cirrhotic portal hypertension," World Journal of Gastroenterology, vol. 11, no. 16, pp. 2513-2517, 2005.

[2] A. J. McMahon, I. T. Russell, J. N. Baxter et al., "Laparoscopic versus minilaparotomy cholecystectomy: a randomized trial," Lancet, vol. 343, no. 8890, pp. 135-138, 1994.

[3] S. L. Zacks, R. S. Sandler, R. Rutledge, and R. S. Brown Jr, “A population-based cohort study comparing laparoscopic cholecystectomy and open cholecystectomy," The American Journal of Gastroenterology, vol. 97, no. 2, pp. 334-340, 2002.

[4] F. Keus, H. G. Gooszen, and C. J. van Laarhoven, "Open, small-incision, or laparoscopic cholecystectomy for patients with symptomatic cholecystolithiasis. An overview of Cochrane Hepato-Biliary Group reviews," The Cochrane Database of Systematic Reviews, no. 1, article CD008318, 2010.

[5] K. M. Harboe and L. Bardram, "The quality of cholecystectomy in Denmark: outcome and risk factors for 20,307 patients from the national database," Surgical Endoscopy, vol. 25, no. 5, pp. 1630-1641, 2011.

[6] J. A. Shea, M. J. Healey, J. A. Berlin et al., "Mortality and complications associated with laparoscopic cholecystectomy. A meta-analysis," Annals of Surgery, vol. 224, no. 5, pp. 609-620, 1996.

[7] A. Agrusa, G. Romano, G. Frazzetta et al., "Role and outcomes of laparoscopic cholecystectomy in the elderly," International Journal of Surgery, vol. 12, Supplement 2, pp. S37-S39, 2014.

[8] V. L. Harrison, J. P. Dolan, T. H. Pham et al., "Bile duct injury after laparoscopic cholecystectomy in hospitals with and without surgical residency programs: is there a difference?" Surgical Endoscopy, vol. 25, no. 6, pp. 1969-1974, 2011.

[9] M. Rosen, F. Brody, and J. Ponsky, "Predictive factors for conversion of laparoscopic cholecystectomy," American Journal of Surgery, vol. 184, no. 3, pp. 254-258, 2002.

[10] B. Tang and A. Cuschieri, "Conversions during laparoscopic cholecystectomy: risk factors and effects on patient outcome," Journal of Gastrointestinal Surgery, vol. 10, no. 7, pp. 1081-1091, 2006. 
[11] B. I. Lengyel, M. T. Panizales, J. Steinberg, S. W. Ashley, and A. Tavakkoli, "Laparoscopic cholecystectomy: what is the price of conversion?" Surgery, vol. 152, no. 2, pp. 173-178, 2012.

[12] S. W. Low, S. G. Iyer, S. K. Chang, K. S. Mak, V. T. Lee, and K. Madhavan, "Laparoscopic cholecystectomy for acute cholecystitis: safe implementation of successful strategies to reduce conversion rates," Surgical Endoscopy, vol. 23, no. 11, pp. 2424-2429, 2009.

[13] J. Ayerdi, J. Wiseman, S. K. Gupta, and S. C. Simon, “Training background as a factor in the conversion rate of laparoscopic cholecystectomy," The American Surgeon, vol. 67, no. 8, pp. 780-785, 2001.

[14] M. Ballal, G. David, S. Willmott, D. J. Corless, M. Deakin, and J. P. Slavin, "Conversion after laparoscopic cholecystectomy in England," Surgical Endoscopy, vol. 23, no. 10, pp. 2338-2344, 2009.

[15] V. H. Le, D. E. Smith, and B. L. Johnson, "Conversion of laparoscopic to open cholecystectomy in the current era of laparoscopic surgery," The American Surgeon, vol. 78, no. 12, pp. 1392-1395, 2012.

[16] J. S. Randhawa and A. K. Pujahari, "Preoperative prediction of difficult lap chole: a scoring method," The Indian Journal of Surgery, vol. 71, no. 4, pp. 198-201, 2009.

[17] M. P. Schijven and J. Jakimowicz, "The learning curve on the Xitact LS 500 laparoscopy simulator: profiles of performance," Surgical Endoscopy, vol. 18, no. 1, pp. 121-127, 2004.

[18] H. M. Kaafarani, T. S. Smith, L. Neumayer, D. H. Berger, R. G. Depalma, and K. M. Itani, "Trends, outcomes, and predictors of open and conversion to open cholecystectomy in veterans health administration hospitals," American Journal of Surgery, vol. 200, no. 1, pp. 32-40, 2010.

[19] U. F. Giger, J. M. Michel, I. Opitz et al., "Risk factors for perioperative complications in patients undergoing laparoscopic cholecystectomy: analysis of 22,953 consecutive cases from the Swiss Association of Laparoscopic and Thoracoscopic Surgery database," Journal of the American College of Surgeons, vol. 203, no. 5, pp. 723-728, 2006.

[20] R. Lavy, A. Halevy, and Y. Hershkovitz, "The effect of afternoon operative sessions of laparoscopic cholecystectomy performed by senior surgeons on the general surgery residency program: a comparative study," Journal of Surgical Education, vol. 72, no. 5, pp. 1014-1017, 2015.

[21] S. Ibrahim, K. H. Tay, S. H. Lim, T. Ravintharan, and N. C. Tan, "Analysis of a structured training programme in laparoscopic cholecystectomy," Langenbeck's Archives of Surgery, vol. 393, no. 6, pp. 943-948, 2008.

[22] J. S. Abelson, C. Afaneh, B. S. Rich et al., "Advanced laparoscopic fellowship training decreases conversion rates during laparoscopic cholecystectomy for acute biliary diseases: a retrospective cohort study," International Journal of Surgery, vol. 13, pp. 221-226, 2015.

[23] R. P. Sutcliffe, M. Hollyman, J. Hodson et al., "Preoperative risk factors for conversion from laparoscopic to open cholecystectomy: a validated risk score derived from a prospective U.K. database of 8820 patients," HPB: The Official Journal of the International Hepato Pancreato Biliary Association, vol. 18, no. 11, pp. 922-928, 2016. 


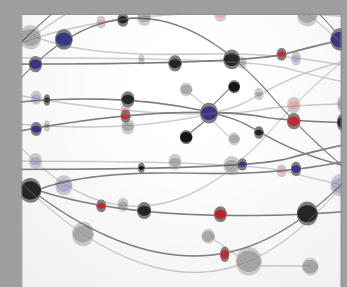

The Scientific World Journal
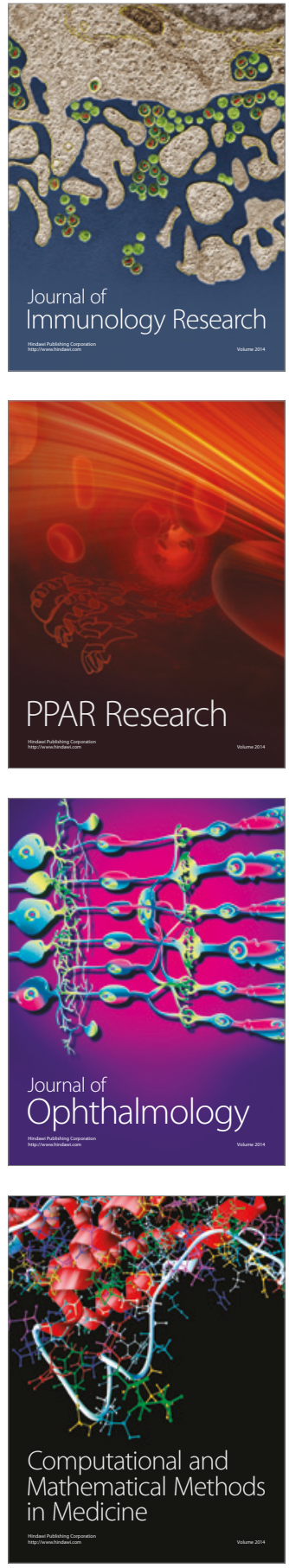

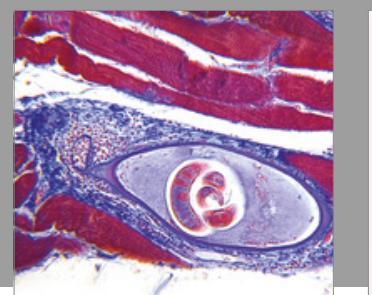

Gastroenterology Research and Practice
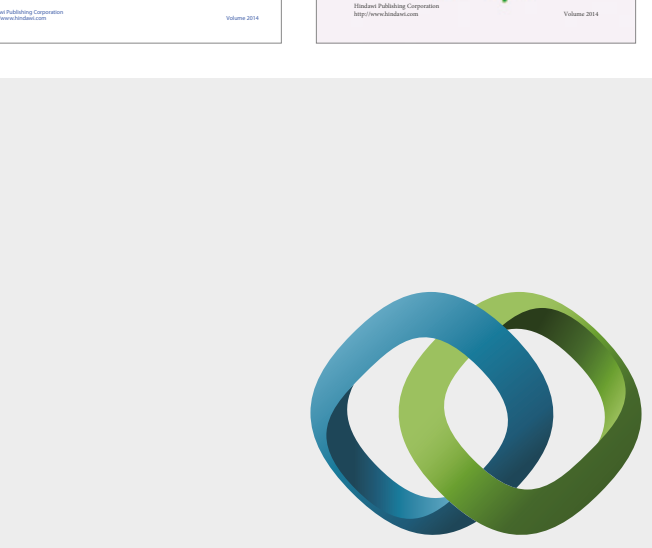

\section{Hindawi}

Submit your manuscripts at

https://www.hindawi.com
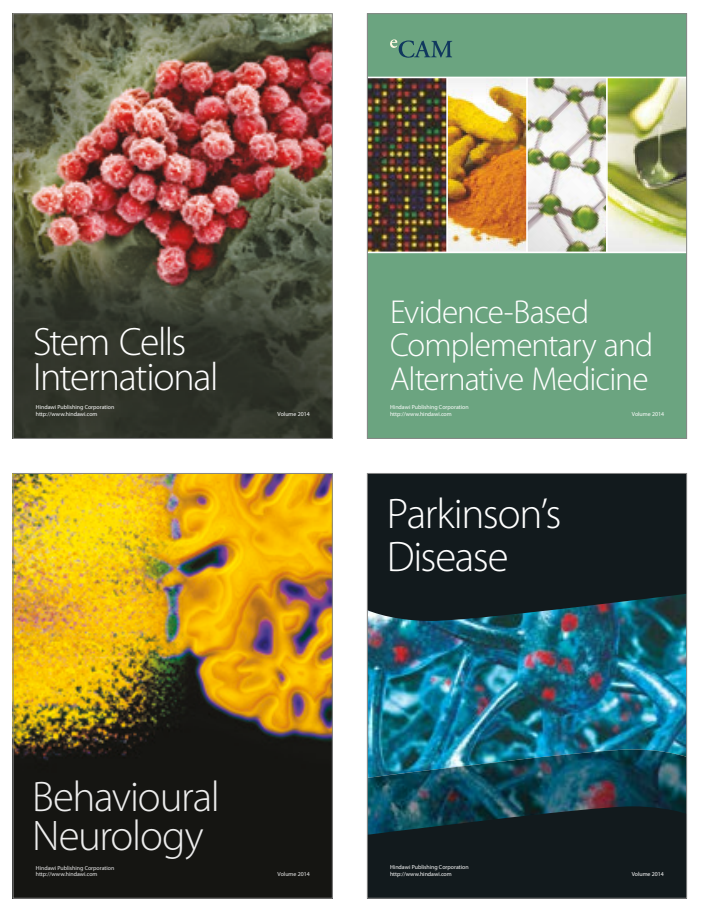
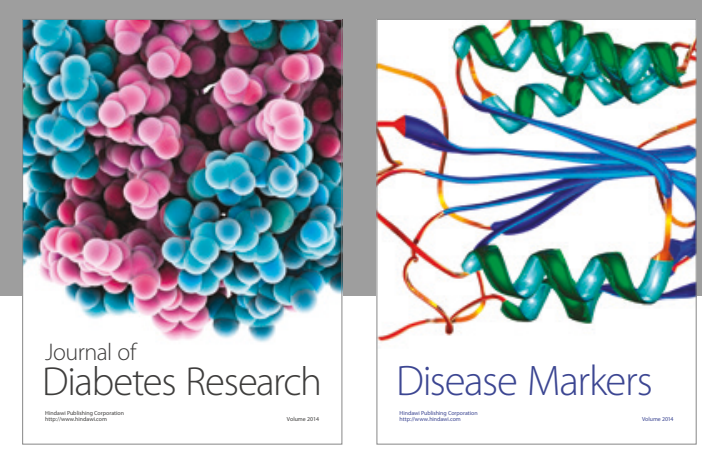

Disease Markers
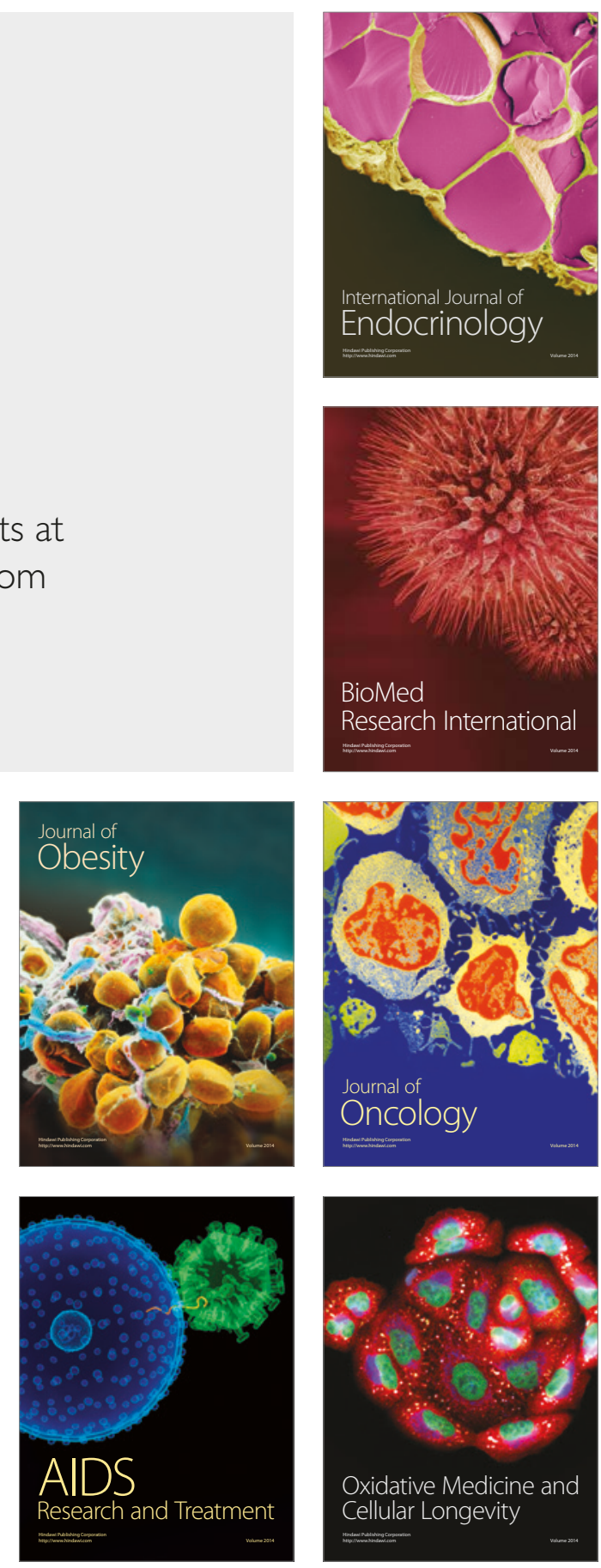